\title{
Biological Materials: Green Source Towards Silver Nanomaterials Synthesis
}

\author{
AMRITA THAKUR* and S. GIRIDHAR REDDY \\ Chemistry Department, Amrita School of Engineering, \\ Amrita Vishwa Vidyapeetham, Bangalore, Karnataka-560035, India. \\ ${ }^{*}$ Corresponding author E-mail: t_amrita@ blr.amrita.edu \\ http://dx.doi.org/10.13005/ojc/320320
}

(Received: May 06, 2016; Accepted: June 18, 2016)

\begin{abstract}
The synthesis of silver nanoparticles is of great importance because of the fact that their application is sensitive to the shape, size and its distribution. Bio medical application also demands that nanoparticles should be free from contaminants. Quest for synthetic methods which are benign to both human and environment led researchers to use locally available biogenic products for silver nanoparticle synthesis. Present review article, is based on the available literature on the use of microorganisms and plants for silver nanoparticle synthesis. Also the factors affecting the synthesis, role of phyto chemicals and proposed application of synthesized nanoparticles have been reported.
\end{abstract}

Keywords: Silver nanoparticles, micro-organisms, plants, phytochemicals.

\section{INTRODUCTION}

Nanotechnology is the manipulation of particles with at least one of its dimension, in the range of 1 to $100 \mathrm{~nm}$. At this dimension a transition in properties from atom or molecule to bulk takes place and their properties too become unique, which often depends on size. For example, bulk semiconductor become insulator at nano scale, noble metal show good catalytic property, some of the metals show colour, luminescence etc. The unique nature of nano particles (NP) is due to the presence of significant number of atoms or ions on the surface and discreteness of energy levels.
Silver nanoparticles (AgNP), due to their unique thermal, optical, electrical, chemical and biological properties in the nano range are studied extensively for application in various fields. Its application in fluorescence based bio sensing ${ }^{1}$ is based on the fact that the band gap, which is discrete at nanometre range, widens further with decrease in size and is reflected as a blue shift in the threshold wavelength. Optical property of AgNPs is also explored in colorimetric detection of chemicals in the environment like triethyl amine ${ }^{2}$, Mercury ${ }^{3}$ etc. Presence of more number of atoms on surface have been helpful in successful application of AgNP as catalysts ${ }^{4}$. They have also been used 
to fabricate photocurable conductive adhesives for application in electronics due to their good conducting properties ${ }^{5}$. Its antibacterial property combined with biocompatibility with human cell has been exploited to incorporate AgNP into applications such as dental work, catheters, bone cement, burn and wound dressing formulations ${ }^{6-7}$, anti-bacterial fabrics $^{8-9}$ etc .Many theories have been proposed for the antimicrobial activity of AgNP. Studies by Ahrland et al and others suggest that silver tends to have a greater affinity with phosphorus and sulphur compounds ${ }^{10-11}$. AgNP of size 1-60 nm have been found to effectively react with sulphur containing proteins inside the cell, as well as phosphorus containing compounds such as DNA in the cell nucleus $^{12}$. Due to smaller size the AgNP enter into the bacterial cell and inactivate bacterial enzymes essential for their metabolic activity, generating hydrogen peroxide, and causing bacterial cell to death ${ }^{13-14}$. The reactions of AgNP with DNA chain lead to its loss of ability to replicate and the cellular proteins of microbe become inactive on silver ion treatment ${ }^{15}$. Silver ions in higher concentration are found to react with cytoplasmic components and nucleic acids ${ }^{16}$ which affect the metabolic process like cell respiration and cell division of bacteria.

Thus, synthesis of AgNP with suitable functionality and of controlled size, shape and the distribution of size and shape is important for successful application. With current environmental burden due to existing industrial processes, it is desirable that the synthetic methods should be sustainable also.

The synthetic approaches are top down and bottom up. Top down approach like ball milling involves breaking of the bulk material into nanometre size range. Though the method is simple and can be easily scaled up, imperfect surface structure is one of the major disadvantages of this method. Bottom up approach on the other hand involves assembling of building blocks like atoms, molecules, and clusters one by one into nano materials. Methods like plasma arching, evaporation condensation etc are examples of bottom up apporoach. These mehhods are direct, can successfully generate mono dispersed and uncontaminated NPs but consume more time and power and also require special setup. Chemical methods are bottom up method and involve reduction followed by agglomeration of molecules/ atoms into oligomeric clusters of nanometre size range. Apart from the precursors, this method needs solvents, reducing agents, and stabilizers which are often harmful to the humans and environment. Contamination of NPs is another problem associated with this method and limits the use of synthesized NPs into biomedical field.

Increasing demand and a quest for a novel method based on the Principles of Green Chemistry resulted into the use natural materials like microorganisms (yeast, fungi, algae, bacteria, virus etc), plants and its products (fruits, fruit peel, bark, seeds) etc as a suitable agent for NP synthesis. These synthetic methods are based on the use of non-toxic chemicals and environment friendly procedures ${ }^{17}$. These methods consume less energy, time and are environmentally benign. As biological methods are chemical methods of synthesis, in general the synthesis is done by reducing silver ion to zero valent silver with the help help of biological species of biological species. In some cases this reduction takes place inside the cell of microorganism. The atomic silver then assembles into NPs. The presence of biomolecules like enzymes, proteins, flavinoids, terpenoids, sugars, phenols etc in the natural products enable them to act as reducing, capping and stabilizing agents for metal NPs. The ability of biomaterials to synthesize NPs is due to the presence of biomolecules responsible for carrying out life processes. These chemicals have the ability to reduce metal ions and stabilize the NPs. The dissimilatory ferric reductase, found in bacteria (mostly intracellular), have been found to be an essential part of the iron cycles ${ }^{18}$. Some bacteria are found to reduce $\mathrm{Fe}^{3+}$ oxides by producing and secreting small, diffusible redox compounds that can serve as electron shuttle between the microbe and the insoluble iron substrate ${ }^{19}$. The role of excreted compounds in extracellular electron transfer has also been recently reviewed in NP synthesis ${ }^{20}$. AgNPs through oligopeptides catalysis, have been found to precipitate in several forms (hexagonal, spherical and triangular $)^{21}$ Goswami et $\mathrm{al}^{22}$ in their study highlighted the role of proteins as stabilizing agent for the synthesized AgNP. Duran et $\mathrm{a}^{23}$ in their attempt to understand the mechanistic aspect of synthesis of AgNP from extracellular extract of Fusarium oxysporum strains found that the reduction of the 
silver ions happens by a nitrate-dependent reductase and a shuttle quinone extracellular process. Roy $\mathrm{M}$ et $\mathrm{a}^{\mathrm{R}}$ has also reported that cysteine present in the cell-free extract from Trichoderma asperellum act as a potential reducing and capping agent in the synthesis of stable AgNPs. Plant extracts also contain metabolites such as polyphenols/ flavonoids, proteins, terpenoids, tannins, etc. ${ }^{25}$. These metabolites not only acts as reducing agents for metal ion but also act as capping agents, which reduces the agglomeration of $\mathrm{NPs}^{26}$.

Present review is based on the recent development of biological methods as a green route for the synthesis of AgNP. Most of the methods are simple, have used locally available biological materials like bacteria, algae, fungi and plants. Steps involved in the synthesis can be summarized in figure1. As reported by the authors, the role of phytochemicals in the NP synthesis, reaction conditions and factors that control rate of synthesis, shape and size of synthesized AgNPs, their morphology etc have also been presented. The possible application of thus synthesized NPs as reported in the literature has also been mentioned in the review.

\section{Micro-organisms and AgNP synthesis}

There are two approaches - Intracellular synthesis which involves the reduction of metal ion and its accumulation as NP on the cell wall, cytoplasmic membrane etc of the microorganism and the extracellular synthesis where the supernatant

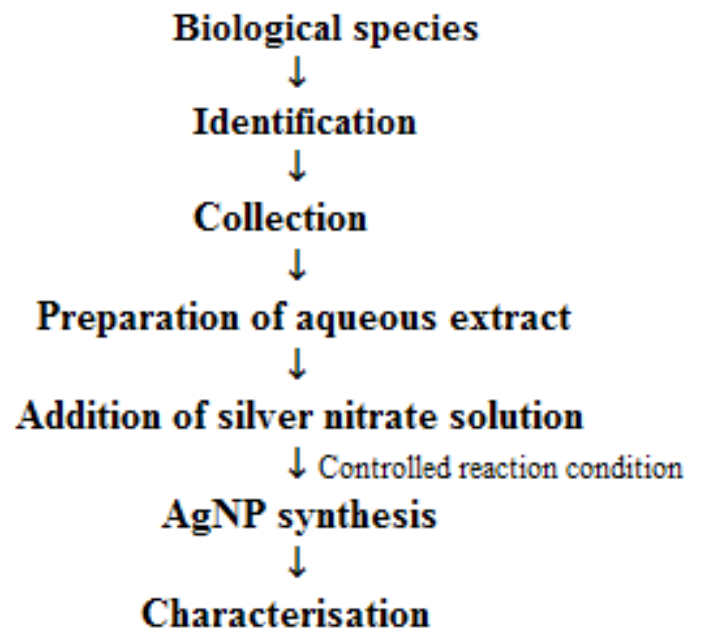

Fig.1: Steps involved in AgNp synthesis obtained from the microbial cell is used for the synthesis of AgNP. To obtain the culture supernatant the microbes are isolated from the growth medium, washed with distilled water to remove the medium completely then incubated for some time. The cell is then filtered out and the filtrate is used as the supernatant. Intracellular synthesis provides a better control over size, shape and distribution of shape but harvesting and recovery of synthesized particles is a major challenge. Recovery of product is easy for extracellular synthesis. Growth of microbe in culture media, waste disposal depending upon the type of microbe used is some of the problems encountered in this approach.

\section{Synthesis of AgNP by Bacteria}

In our literature survey we observed that many locally available bacteria has been explored for the synthesis of AgNP. Crystalline AgNP with fcc (face centred cubic) structure were synthesized when cell extract of photosynthetic bacteria Rhodobacter Sphaeroides and silver nitrate solution were exposed to light of intensity 2500 lux at $30^{\circ} \mathrm{C}$. Time taken for synthesis was 6 hours. Size of AgNPs was reported to be controlled by the specific activity of nitrate reductase in the cell filtrate ${ }^{27}$. A novel strain of Bacillus safensis with potent keratinolytic activities (KA) was isolated in a study for the biotechnological management of feather wastes and creation of novel products. Cell eatract of this bacteria was investigated for its ability to produce AgNPs and evaluate its applications. In this case crystalline, spherical and fcc structured AgNP of the size 5 - 30 $\mathrm{nm}$ were obtained. Protein was reported to act as a capping and stabilizing agent The synthesized particles showed good anti-bacterial activity against E. coli ${ }^{28}$. Microbes may develop nano particle synthesis ability when exposed continuously to matals for a long period of time. Bacterial strain (CS 11) isolated from heavy metal contaminated soil, on treating with $1 \mathrm{mM} \mathrm{AgNO}_{3}$, was found to have the ability to form AgNPs extracellularly at room temperature within $24 \mathrm{~h}$. This was confirmed by the visual observation and UV-Vis absorption at 450 $\mathrm{nm}$. Characterization of NPs by transmission electron microscopy confirmed the size of AgNPs in 42-92 $\mathrm{nm}$ range. Nitrate reductase was proposed to be the reducing agent in this case also ${ }^{29}$. A rapid synthesis of AgNP was achieved when cells of Haloferax alexandrinus were exposed to silver nitrate solution 
under direct sunlight. The synthesized NP showed good anti-microbial property ${ }^{30}$. AgNP, average size $26 \mathrm{~nm}$, were also synthesized in the cells of silver tolerant marine bacteria Idiomarina sp. PR58-8 in 42 hrs. The high concentration of non-protein thiol in the metal exposed bacterium suggests the sequestration of AgNP by it ${ }^{31}$. Rhodococcus bacterial strains are present in wide range of habitats, has industrial applications like biotransformation, biodegradation of organic compounds etc. AgNP are synthesized in one of the study from the cell extract of these bacteria obtained from the supernatant of phenol degradation broth. NP, thus obtained were found to be stable over wide range of $\mathrm{pH}$ and temperature which is attributed to the electrostatic repulsion of negative charge present on the protein molecules on its surface. The above synthesized NP display good antimicrobial activity against both gram negative and gram positive bacteria and also good catalytic activity for the reduction of 4-Nitro Phenol by $\mathrm{NaBH}_{4}^{32}$.

\section{Synthesis of AgNP by Fungi}

Actinomycetes are microorganism having characteristic of both fungi and bacteria. Its extract was used to synthesize AgNP of $198-595 \mathrm{~nm}$ size in dark. The synthesized particles showed good antimicrobial property for gram negative bacteria and moderate for gram positive bacteria. The synthesized AgNP showed a very good cytotoxicity against A549 adenocarcinoma the lung cancer cell line ${ }^{33}$. Extracellular biosynthesis of AgNPs was achieved by Epicoccum nigrum, an endophytic fungus isolated from the cambium of Phellodendron amurense. The synthesized AgNPs were exceptionally stable, spherical and were in the size range of 1 to $22 \mathrm{~nm}$. It was found that an alkaline $\mathrm{pH}$ favored the formation of AgNPs and elevated temperature accelerated the reduction process. Furthermore, the antifungal activity of the AgNPs was assessed using a microdilution method. The biosynthesized AgNPs showed considerable activity against the pathogenic fung ${ }^{34}$. Stable NP over wide range of ionic strength, $\mathrm{pH}$ and temperature have been synthesized using cell free filtrate of Ascomycota Fungi Penicillium nalgiovense AJ12. Presence of protein molecules act as reducing and capping agent ${ }^{35}$. Another study, AgNP was synthesized using Neurospora intermedia. Study indicated highest productivity with smallest size of NP obtained when equal volume of culture supernatant and silver nitrate was mixed, while the NP of highest monodispersity were obtained when the reaction was carried out in dark. The rate of reaction was found to be slow. The role of nitrate reductase in the synthesis was proposed ${ }^{36}$. Mushroom Fungus Schizophyllum commune could successfully synthesize AgNP extracellularly as well as intra cellularly in neutral solution at ambient temperature. Synthesized particles showed excellent anti-microbial and anti dermatophytic activity. The synthesized particles also showed good anti-cancer activity for Human Epidermoid Larynx Carcinoma (HEP -2) cells ${ }^{37}$. Extracellular synthesis of AgNP of average size $14.5 \mathrm{~nm}$ was achieved by Schizophyllum radiatum exract. The effect of carbon source on the synthesis was also studied. Best result was obtained with glucose and fructose which was explained by the fact that these carbon sources enhance the synthesis of enzymes responsible for reduction of metal ions. Above synthesized metal particles showed good antimicrobial property ${ }^{38}$. In a similar experiment polydispersed, spherical,protein stabilized AgNP with an average diameter of $16.23 \mathrm{~nm}$ were synthesized by extracellular extract of Fusarium solani, a plant pathogen ${ }^{39}$. Another synthetic method was developed using extracellular extract of Beauveria bassiana, an entomopathogenic fungus. The NP was found to be an effective larvicidal agent against Aedes aegypti, the dengue vector ${ }^{40}$.

\section{Synthesis of AgNP by Algae}

Extract of Caulerpa racemosa an algae from sea has been used to synthesize AgNP of the size 5-25 nm at room temperature. Role of peptides in the synthesis was suggested. Synthesized NP showed good anti-bacterial activity against Staphylococcus aureus and Proteus mirabilis ${ }^{41}$. In another study spherical, crystalline, AgNP, size 5-15 nm were synthesized from sea algae Sargassum muticum in aqueous medium. Studies indicated sulphated polysaccharides as the reducing agents ${ }^{42}$. AgNP of average size $20 \mathrm{~nm}$ were synthesized by heating at $60^{\circ} \mathrm{C}$, in water bath when aqueous extract of three types of sea weeds Sargassum plagiophyllum, Ulva reticulata and Enteromorpha compressa, were reacted separately with silver nitrate solution. Sargassum plagiophyllum was found to have better synthetic ability amongst the three ${ }^{43}$. Sargassum longifolium an algae from sea was explored for AgNP synthesis and the effect of time and $\mathrm{pH}$ for the synthesis was studied. Observations showed 
that the synthesis was favoured at higher $\mathrm{pH}$ and longer reaction period. The polydispersed and spherical NP showed a good anti-fungal property against Aspergillus fumigatus , Candida albicans, Fusarium $s p^{44}$.

\section{Synthesis of AgNP by Plants}

Plant mediated synthesis have been reported in many literatures and are presently gaining importance ${ }^{45}[44 \mathrm{~A}]$. Aqueous extract of whole plant, leaf, root, latex, seed, bark, fruit etc have been used. Phytochemicals present in extract are found to be responsible for the synthesis. Ease of preparation of plant extract, simple synthetic procedures, and scalability are some of the advantages with plants. In most of the studies, locally available plant species have been explored. In one of the study AgNP was synthesized by autoclaving silver nitrate solution with Anogeissus latifolia(gum ghatti). Effect of concentration of gum and reaction time on NP synthesis was reported. Proposed mechanism suggest that the $\mathrm{Ag}^{+}$ion first gets attached to the hydroxyl and carboxylic acid group present on the polymer, then oxidises the hydroxyl group and thus self gets reduced to metallic silver. Also some aldehydic groups formed due to oxidation with air already present in reaction mixture and other carbonyl groups on polymer play an important role in the reduction of silver ion. The NP then formed are stabilized by polysaccharides and proteins of the gum. Synthesized particles showed good anti-microbial property ${ }^{46}$. Crystalline AgNP of average size $17 \mathrm{~nm}$ were obtained by irradiating seed extract of sweet acacia and silver nitrate solution with microwave radiation for 150s. Presence of flavonones and terpenoids on the surface of metal NP were confirmed by ${ }^{13} \mathrm{C} \mathrm{nmr}$. The synthesized NP showed good antimicrobial property ${ }^{47}$.

Cissus quadrangularis, a perennial climber, is used in many ayurvedic formulations. Crystalline AgNP of size between 37-43 nm were synthesized at room temperature by reacting its aqueous extract with silver nitrate. The effect of silver nitrate concentration, $\mathrm{pH}$, reaction time and temperature on the synthesis was studied. High temp, $\mathrm{pH}$, and a longer reaction period were found to favour NP synthesis ${ }^{48}$. Phyllanthusniruri, a hepatoprotective plant was also used to synthesize AgNP. To prepare plant extract, leaf powder was incubated with water for $48 \mathrm{hrs}$ and the supernatant was collected and dried by evaporation. Extract of different concentration was prepared by taking different weights of the dry powder. AgNP were synthesized by mixing the extract with silver nitrate ${ }^{49}$. In another study bio extract of citrus lemon was prepared by boiling leaf pieces in water for 5-10 $\mathrm{min}$. The filtrate obtained was treated with silver nitrate at room temperature in dark. The synthesis of NP was observed after one hr. Synthesis of heterogeneous, well dispersed NP stabilized by plant chemicals like flavones, terpenoids, polysaccharides, proteins was reported. Thus prepared NP were deposited on cotton and silk fabrics. The textile finish was found to be durable in both the cases. Also the modified textiles were reported to show a good antifungal property against Fusarium oxysporum and Alternaria brassicicola $^{50}$. Ocimum sanctum has been known for its medicinal properties. In one of the study its extract was prepared by stirring the leaf in water at $60^{\circ} \mathrm{C}$ for one $\mathrm{hr}$. The extract was reported to synthesize crystalline, spherical AgNP of average size $14.31 \pm 2.5 \mathrm{~nm}$ within 8 minutes at $30^{\circ} \mathrm{C}$. FTIR studies suggest the stabilization of NP by proteins. The NP thus synthesized were also reported to show good antimicrobial peoperty ${ }^{51}$. Another study reported synthesise of crystalline AgNP when aqueous extract of allium cepa and silver nitrate was exposed to microwave radiation. The synthesis rate was found to increase with irradiation time and power. Also smaller NP were synthesized when reaction time was increased. A good antimicrobial activity against gram negative bacteria Escherichia coli was observed. The possible reason suggested was the presence of thin peptidoglycan layer in these types of bacteria as compared to gram positive one ${ }^{52}$. Aqueous leaf extract of mangosteen was used for NP synthesis and various reaction parameters were studied. Optimum synthesis was observed at $75{ }^{\circ} \mathrm{C}$, neutral $\mathrm{pH}, 60 \mathrm{~min}$. of reaction time, $1 \mathrm{mM}$ $\mathrm{AgNO}_{3}$ concentration and a 1:19 $\mathrm{AgNO}_{3}$ plant extract mixture. The NP's were found to be stable for 30 days. Good antibacterial property for these NP was also reported ${ }^{53}$.

Synthesis of AgNP was also reported from plant powders of Solanum tricobatum, Syzygium cumini, Centella asiatica and Citrus sinensis. Extracts were prepared by soaking the powders in alcohol, then drying the alcoholic supernatant by 
evaporation. The dry powder was then dissolved in water to prepare the aqueous extract. Synthesized NP were reported to show good anti-microbial property ${ }^{54}$. Silver and gold NP were synthesized from fruit extract of Prunus armeniaca. The synthesis was favoured at higher $\mathrm{pH}$. Also the particles were found to show good in vitro scavenging activity for free radicals $\mathrm{DPPH}$ and $\mathrm{ABTS}$ with a higher sensitivity for $\mathrm{ABTS}^{55}$. In a unique approach, AgNP were synthesized using leaf extract of Manilkara zapota. When these NP were exposed to adult house fly, $100 \%$ mortality was reported within three $\mathrm{hrs}^{56}$. It is important to control of size of nano particles for a wide range of applications. The squama inner coat of onion has been used as a biological template to synthesize monodisperse AgNP in 4.6 to $7.9 \mathrm{~nm}$ size range with an fcc structure. Ascorbic acid was used as reducing agent and the synthesis time was $8 \mathrm{hrs}$. The study also indicated that the morphology of AgNP is dependent on the concentration of both silver nitrate and ascorbic acid. A better result was observed with low concentration and synthesis could not be achieved beyond a concentration of $0.4 \mathrm{~mol} / \mathrm{l}$ of the reactants. Also the study indicated a moderate temperature of $25-30^{\circ} \mathrm{C}$ suitable for the synthesis. It is suggested that the silver ions first accumulate on the grooves and holes on the surface of squama inner coat due to the attraction between positive charge on the silver and the negatively charged hydroxyl and carboxylate groups. There the reductions with carboxylate ions produce silver atoms. Then forms the nucleus which grows in size. The growth of various faces is controlled by the templating groups ${ }^{57}$. Strychnos potatorum is a medicinal plant used for treatment of wide range of disease like bacterial infections, urinary tract infections, kidney problems etc. Aqueous cell free extract of air dried leaf of this plant was added to aqueous solution of silver nitrate precursor in a rotary orbital shaker at $150 \mathrm{rpm}$ for $25 \mathrm{hrs}$ at $25^{\circ} \mathrm{C}$ in dark. Spherical NP of $16-18 \mathrm{~nm}$ were synthesized. The NPs were found to be stable and showed good antibacterial properties against gram positive and gram negative bacteria $S$. aureus and $K$. pneumonia respectively ${ }^{58}$. In an attempt to synthesize AgNPs bark of a local tree (Afzelia quanzensis) from Zimbabwe was used. The bark was dried, powedered, and then boiled with deionized water to prepare the aqueous extract. The extract was then used to reduce the silver ions to prepare the AgNPs. The synthesized particles were of 10$80 \mathrm{~nm}$ and were found to be stable for 40 days in water ${ }^{59}$. antimicrobial activity against fungal as well as bacterial cultures were also observed from AgNP synthesized by incubating silver nitrate solution and aqueous extract of Pistacia lentiscus leaves at $50^{\circ} \mathrm{C}^{60}$.

\section{CONCLUSION}

Biological methods involving locally available natural products like plants, fungi, algae, bacteria etc are successful in synthesizing crystalline polydispersed AgNP of size below $100 \mathrm{~nm}$ with least of experimental setup. Water has been used as experimental medium and synthesis could be achieved under ambient conditions. Role of phyto chemicals in the synthesis and stabilization of NP have been established. More experiments are reported with plants than fungi, algae and bacteria, possible reason could be the simplicity of synthetic procedure involving plants. Synthesized AgNP have been explored successfully as antimicrobial agent. Little has been reported about the mechanism of NP synthesis. These processes can be used for large scale production of AgNP in future.

\section{REFERENCES}

1. Wenw Nan Zhong, Anal Bioanal Chem, 2009, 94, 47-59.

2. Filippoa, E.; Mannob, D.; Buccolierib, A.; Serra, A, Sensors and Actuators B. Chemical, 2013, 178, 1- 9.

3. Shuyan Gao.; Xiaoxia Jia.; Yanli Chen, J Nanopart Res, 2013, 15, 1385.

4. Yasmeen Junejo.; Baykal, A.; Sirajuddin, $J$ Inorg Organomet Polym, J Inorg Organomet
Polym, 2014, 24, 401-406.

5. Wen-Tung Cheng.; Yu-Wen Chih.; Wei-Ting Yeh, International Journal of Adhesion \& Adhesives, 2007, 27, 236-243.

6. Catauro, M.; Raucci, M.G.; De Gaetano, F.D.; Marotta, A, J Mater Sci Mater Med, 2004, 15(7), 831 - 7.

7. Crabtree, J.H.; Burchette, R.J.; Siddiqi, R.A.; Huen, I.T.; Handott, L.L.; Fishman, A, Perit 
Dial Int, 2003, 23(4), 368- 74.

8. Tian Jiang.; Lin Liu,; Juming Yao, Fibers and Polymers, 2011, 12(5), 620-625.

9. Bin Tang.; Jasjeet Kaur.; Lu Sun.; Xungai Wang, Cellulose, 2013, 20(6), 3053-3065.

10. Ahrland, S.; Chatt, J.; Davies, N.R, Q Rev Chem Soc, 1958, 12, 265-276.

11. Hatchett, D.W.; Henry, S, J Phys Chem, 1996, 100, 9854-9859.

12. Prabhu, S.; Poulose, E.K, Int Nano Lett, 2012, 32, 1-10.

13. Stockman, G.D.; Barrett, J.F, Annu Rev Microbiol, 1983, 37:501-527.

14. Raffi, M.; Hussain, F.; Bhatti, T.M.; Akhter, J.I.; Hameed, A.; Hasan, M.M, , J Mater Sci Technol, 2008, 24, 192-196.

15. Kumar, A.; Kumar Vemula, P.; Ajayan, P.M.; John, G. Nat Mater, 2008, 7, 236-241.

16. Feng, Q.L.; Wu, J.; Chen, G.Q.; Cui, F.Z., Kim, T.N.; Kim, J.O, J Biomed Mater Res, 2000, 52, 662-668.

17. Anastas., P.T.; Warner, J.C, Green Chemistry: Theory and Practice, 1998 New York: Oxford University Press, Inc.

18. Schroder, I.; Johnson, E.; De Vries, S, FEMS, Microbiol Rev, 2003, 27, 427-447.

19. Newman, D.K.; Kolter, R, Nature, 2000, 405, 94-97.

20. Hernandez, M.E.; Newman, D.K:, Cell Mol Life Sci, 2001, 56, 1562-1571.

21. Naik, R.R.; Stringer, S.J.; Agarwal, G.; Jones, S.E.; Stone, M.O, Nat Mater, 2002, 1, 169172.

22. Goswami .,AMB Express. 2013, 3, 16.

23. Nelson Durán.; Priscyla, D.; Marcato.; Oswaldo, L.; Alves.; Gabriel, I.H.D.; Souza.; Elisa Esposito, Journal of Nanobiotechnology, 2005, 3, 8.

24. Roy, M.; Mukherjee, P.; Mandal, P.; Sharma, K.; Tyagi, K.; Kale, P, RSC Advances, 2011, 2, 6496-6503.

25. Raghunandan, D.; Mahesh, B. D.; Basavaraja, S.; Balaji, S. D.; Manjunath, S. Y.; Venkatraman, A, J. Nanopart. Res, 2011, 13, 2021.

26. Haung, J.; Sun, Q; Li, D.; Su, Y. Lu.; Yang, X.; Wang, H.; Wang, Y.; Shao, W.; He, Hong, J.N.; Chen, C, Nanotechnology, 2007, 18, 104.

27. Hong Juan Bai.; Bin Sheng.; Yang.; Chun Jing Chai.; Guan, E.; Yang.; Wan Li Jia.; Zhi Ben, Yi, World Journal of Microbiology and
Biotechnology, 2011, 27, 2723.

28. Lateef, A.; Adelere, I.A.; Gueguim Kana, E.B.; Asafa,'T.B.; Beukes, T.B, International Nano Letters, 2015, 5(1), 29-35.

29. Vidhya Lakshmi Das.; Roshmi Thomas.; Rintu, T. Varghese.; Soniya, E. V.; Jyothis Mathew.; Radhakrishnan, E.K, Biotech, 2014, 4(2), 121-126.

30. Sushama Patil .; Julio Fernandes.; Rudreshwar Tangasali.; Irene Furtado, 2014, J Clust Sci, 25(2), 423-433.

31. Sachin seshadri.; Anupama Prakash.; Meenal kowshik, Bull. Mater. Sci., 2012, 35(7), 1201-1205.

32. Otari, S. V.; Patil, R. M.; Nadaf, N. H.; Ghosh, S.J.; Pawar, S.H, Environ Sci Pollut Res, 2014, 21, 1503-1513.

33. Saravana Kumar, P.; Balachandran, C.; Duraipandiyan, V.; Ramasamy,D.; Ignacimuthu, S.; Naif Abdullah Al-Dhabi., Applied Nanoscience, 2015, 5(2), 169-180.

34. Yongqing Qian.; Huimei,Yu.; Dan He.; Hui Yang .;Wanting Wang .; Xue Wan.; Li Wang, Bioprocess Biosyst Eng, 2003, 36, 16131619.

35. Irena Maliszewska.; Anna Juraszek.; Katarzyna Bielska, Journal of Cluster Science 2013, 25(4), 989-1004.

36. Sepideh Hamedi.; Sayed Abbas Shojaosadati.; Soheila Shokrollahzadeh.; Sameereh Hashemi Najafabadi, World Journal of Microbiology and Biotechnology, 2014, 30(2), 693-704.

37. Ganesan., Arun.; Muthukumarasamy Eyini.; Paramasamy Gunasekaran, Biotechnology and Bioprocess Engineering, 2014, 19, 10831090.

38. Ram Prasad Metuku.; Shivakrishna Pabba.; Samatha Burra.; Hima Bindu.; Krishna, N.; Gudikandula.; Singara Charya, M. A, Biotech, 2014, 4, 227-234.

39. Avinash Ingle.; Mahendra Rai.; Aniket Gade.; Manisha Bawaskar, J Nanopart Res, 2009, 11, 2079-2085.

40. Najitha Banu, A.; Balasubramanian, C, Parasitol Res, 2014, 113, 2869-2877.

41. Kathiraven, T.; Sundaramanickam, A.; Shanmugam, N.; Balasubramanian, T, Applied Nanoscience, 2014, 5(4), 499-504.

42. Susan Azizi.; Farideh Namvar.; Mahnaz 
Mahdavi.; Mansor Bin Ahmad.; Rosfarizan Mohamad, Materials, 2013, 6, 5942-5950.

43. Dhanalakshmi, P.K.; Riyazulla Azeez.; Rekha, R.; Poonkodi, S.; Thangaraju Nallamuthu, Phycological Society, India, Phykos, 2012, 42(2), 39-45.

44. Shanmugam Rajesh kumar.; Chelladurai Malarkodi.; Kanniah Paulkumar.; Mahendran Vanaja.; Gnanadas Gnanajobitha.; Gurus, International Journal of Metals, 2014, Article ID 692643, 8.

45. Varahalarao Vadlapudi.; Kaladhar, D.S.V.G.K.; Mohan Behara.; Sujatha,B.; Kishore Naidu, G. Orient j chem., 2013, 29(4), 1589-1595.

46. Aruna Jyothi Kora.; Sashidhar Rao Beedu.; Arunachalam Jayaraman, Organic and Medicinal Chemistry Letters, 2012, 2, 17.

47. Yallappa, S.; Manjanna, J.; Peethambar, S.K.; Rajeshwara,A.N.; Satyanarayan, N.D.; Annadura, J Clust Sci, 2013, 24(4), 10811092.

48. MahendranVanaja.; Gnanadhas Gnanajobitha.; Kanniah Paulkumar.; Shanmugam Rajesh kumar.; Chelladurai Malarkodi.; Gurusamy Annadurai, Journal Of Nanostructure in Chemistry, 2013, 3, 17.

49. Krishnamoorthy, P.; Jayalakshmi, T, Journal of Chemical and Pharmaceutical Research, 2012, 4(11), 4783-4794.

50. Padma, S.; Vankar.; Dhara Shukla, Appl Nanosci, 2012, 2, 163-168.
51. Garima Singhal.; Riju Bhavesh.; Kunal Kasariya.; Ashish Ranjan Sharma.; Rajendra Pal Singh, J Nanopart Res, 2011 , 13, 29812988.

52. Younes Abboud.; Adil Eddahbi.; Abdeslam, E.; Bouari.; Hafid Aitenneite.; Khalid Brouzi.; Jamal Mouslim, Journal of Nanostructure in Chemistry, 2013, 3, 84.

53. Ravichandran Veerasamy A.; Tiah Zi Xin.; Subashini Gunasagaran.; Terence Foo Wei Xiang.; Eddy Fang Chou Yang.; Nelson Jeyakumar ; Sokkalingam Arumugam Dhanaraj Journal of Saudi Chemical Society, 2011, 15, 113-120.

54. Logeswari,P.; Silambarasan,S.; Abraham, J, Scientia Iranica, 2013, 20(3), 1049-1054.

55. Preeti Dauthal.; Mausumi Mukhopadhyay, J Nanopart Res, 2013, 15, 1366.

56. Chinnaperumal Kamaraj.; Govindasamy Rajakumar.; Abdul Abdul Rahuman.; Kanayairam Velayutham.; Asokan Bagavan .; Abdul Abduz Zahir.; Gandhi Elango, Parasitol Res, 2012, 111, 2439-2448.

57. Ping Chen.; Qing-Sheng Wu.; Ya-Ping Ding, Journal of Nanoparticle Research, 2008, 10, 207-213.

58. Srikanth Kagithoju.; Vikram Godishala :; Rama Swamy Nanna, biotech, 2015, 5, 709714.

59. Mambo Moyo.; Makore Gomba.; Tichaona Nharingo, Int $J$ Ind Chem, 2015, 6, 329-338.

60. Ghadir,A.; El-Chaghaby.; Abeer, F. Ahmad, Orient $j$ chem, 2011, 27(3), 929-936 